\title{
Plasmon spectrum of single-layer antimonene
}

\author{
Guus Slotman, ${ }^{1}$ Alexander Rudenko, ${ }^{2,1}$ Edo van Veen, ${ }^{1}$ Mikhail I. Katsnelson, ${ }^{1}$ Rafael Roldán, ${ }^{3}$ and Shengjun Yuan ${ }^{2,1, *}$ \\ ${ }^{1}$ Institute for Molecules and Materials, Radboud University, Heyendaalseweg 135, 6525AJ Nijmegen, The Netherlands \\ ${ }^{2}$ School of Physics and Technology, Wuhan University, Wuhan 430072, China \\ ${ }^{3}$ Materials Science Factory, Instituto de Ciencia de Materiales de Madrid (ICMM), \\ Consejo Superior de Investigaciones Científicas (CSIC), Cantoblanco E28049 Madrid, Spain
}

(Received 29 June 2018; published 10 October 2018)

\begin{abstract}
The collective excitation spectrum of two-dimensional (2D) antimonene is calculated beyond the low-energy continuum approximation. The dynamical polarizability is computed using a six-orbital tight-binding model that properly accounts for the band structure of antimonene in a broad energy range. Electron-electron interaction is considered within the random phase approximation. The obtained spectrum is rich, containing the standard intraband 2D plasmon and a set of single interband modes. We find that spin-orbit interaction plays a fundamental role in the reconstruction of the excitation spectrum, with the emergence of novel interband branches in the continuum that interact with the plasmon.
\end{abstract}

DOI: 10.1103/PhysRevB.98.155411

\section{INTRODUCTION}

Antimonene, a single layer of $\mathrm{Sb}$ atoms arranged in a buckled honeycomb lattice [1], has been recently fabricated by different methods, from mechanical [2] and chemical exfoliation [3], to epitaxial growth [4-6]. As phosphorene, it is a monoelemental atomically thin crystal of group-VA, with the advantage of being highly stable in ambient conditions. Single-layer $\mathrm{Sb}$ is an indirect gap semiconductor, with a strong spin-orbit coupling (SOC). Compared with standard 3D semiconductors such as gallium arsenide or silicon, twodimensional (2D) materials present many special features like quantum confinement in the direction perpendicular to the layer, tuneability of the band gap, or easy integrability in optoelectronic structures, what make them good candidates for nanophotonics [7].

Huge attention has been paid to the study of plasmons [8] and polaritons [9] of 2D materials like graphene [10-13], black phosphorus [14-16], or hexagonal boron nitride (h-BN) [17]. Antimonene, like other families of 2D materials [18], presents optical and electronic properties that can be manipulated and tuned by controlled thickness (number of layers), applied strain, external electric fields, or chemical functionalization. In particular, while a single layer of antimonene is a trivial semiconductor, increasing the number of layers can lead to a transition to a topological semimetal, including the appearance of quantum spin Hall phases. Furthermore, the strong SOC present in this material combined with its high flexibility means that strain engineering can be used to drive a transition from a trivial indirect gap semiconductor to a $2 \mathrm{D}$ topological insulator $[1,19]$. This may open the door to use antimonene for nanoscale transistors with high on/off ratio, nanomechanical sensors, or optoelectronics devices operating in a broad range of the spectrum.

In this paper, we present a systematic study of the excitation spectrum of single-layer antimonene. The band structure

\footnotetext{
*s.yuan@whu.edu.cn
}

is obtained from a tight-binding (TB) Hamiltonian that considers the 3- $p$ orbitals of each of the two $\mathrm{Sb}$ atoms of the unit cell [20]. The dielectric function is obtained within the random phase approximation (RPA). Due to the large number of bands considered in the calculation, the obtained excitation spectrum is rich, with several plasmon and interband modes. Consideration of SOC is shown to be essential to capture the correct low-energy excitations, due to strong reconstruction of the band structure.

\section{METHOD}

Single-layer antimonene has a buckled honeycomb structure with lattice parameter $a=4.12 \AA$, where the two sublattices are vertically displaced by $b=1.65 \AA$. The band structure, including SOC, can be well described by the TB model developed in Ref. [20]. The TB model Hamiltonian is given by

$$
\mathcal{H}=\sum_{m, n} \sum_{i, j} t_{i j}^{m n} c_{i m}^{\dagger} c_{j n}+\mathcal{H}_{\mathrm{SO}}
$$

where $t_{i j}^{m n}$ is the hopping parameter between the $m$ and $n$ orbitals at sites $i$ and $j$. The model considers the three $p$ orbitals of $\mathrm{Sb}$, the hopping parameters being obtained from the formalism of maximally localized Wannier functions. Fifteen relevant terms are included, corresponding to hopping between atoms separated up to a distance of $8.24 \AA$. Intra-atomic SOC is considered by the term $\mathcal{H}_{\mathrm{SO}}=\lambda \mathbf{L} \cdot \mathbf{S}$, where $\mathbf{L}$ and $\mathbf{S}$ are the total atomic angular momentum operator and the total electronic spin operator, respectively, and $\lambda=0.34 \mathrm{eV}$ is the intra-atomic SOC constant obtained for $\mathrm{Sb}$. The band structure obtained from this TB model, Fig. 1, fairly reproduces the spectrum obtained from first-principles density-functionaltheory methods in an energy range from $[-4,+3] \mathrm{eV}$. We notice the important role played by SOC, that reconstructs the band structure leading to an overall reduction of the indirect gap in $\sim 0.3 \mathrm{eV}$, as well as to a number of avoided crossings 


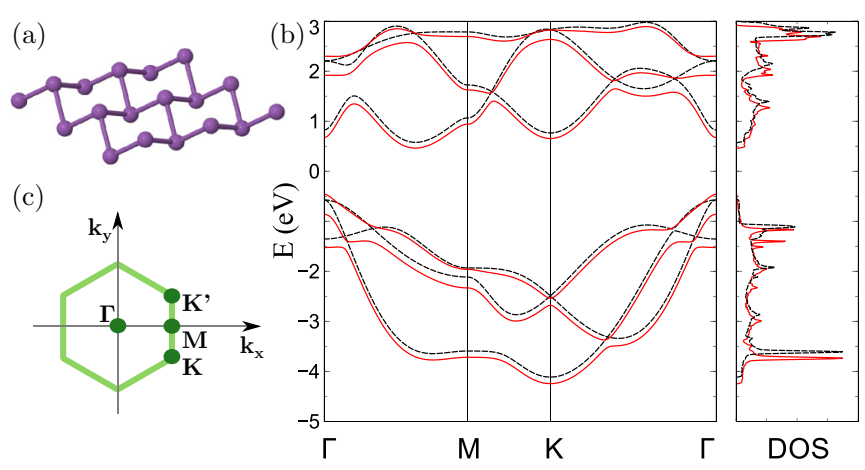

FIG. 1. (a) The buckled atomic structure and (c) Brillouin zone of SL-Sb. (b) The band structure of SL-Sb as obtained in Ref. [20] using their TB Hamiltonian with (solid red lines) and without (dashed black lines) spin-orbit coupling.

between some bands. Those will play an important role when studying the single particle excitation spectrum, as we will see below.

The TB-Hamiltonian Eq. (1) is used to numerically calculate the dynamical polarization $\Pi(\mathbf{q}, \omega)$ and the dielectric function $\epsilon(\mathbf{q}, \omega)$ using the Lindhard function [21]

$$
\begin{aligned}
\Pi(\mathbf{q}, \omega)= & -\frac{g_{s}}{(2 \pi)^{2}} \int_{\mathrm{BZ}} d^{2} \mathbf{k} \sum_{l, l^{\prime}} \frac{n_{F}\left(E_{\mathbf{k} l}\right)-n_{F}\left(E_{\mathbf{k}^{\prime} l^{\prime}}\right)}{E_{\mathbf{k} l}-E_{\mathbf{k}^{\prime} l^{\prime}}+\omega+i \delta} \\
& \times\left|\left\langle\mathbf{k}^{\prime} l^{\prime}\left|e^{i \mathbf{q} \cdot r}\right| \mathbf{k} l\right\rangle\right|^{2},
\end{aligned}
$$

where $|\mathbf{k} l\rangle$ and $E_{\mathbf{k} l}$ are eigenstates and eigenvalues of the Hamiltonian Eq. (1), respectively, with $l$ being the band index, $\mathbf{k}^{\prime}=\mathbf{k}+\mathbf{q}, n_{F}(E)=\frac{1}{e^{\beta(E-\mu)}+1}$ is the Fermi-Dirac distribution, $\delta \rightarrow 0^{+}$and we have taken $\hbar=1$. The integral is taken over the Brillouin zone (BZ), and the sum is calculated over all bands in the TB Hamiltonian. From the dynamical polarization, one can obtain the dielectric function within the RPA as

$$
\epsilon(\mathbf{q}, \omega)=\mathbf{1}-V(q) \Pi(\mathbf{q}, \omega)
$$

with $V(q)=\frac{2 \pi e^{2}}{\epsilon_{B} q}$ the Fourier component of the Coulomb interaction in two dimensions, with $\epsilon_{B}$ being the background dielectric constant. We use $\epsilon_{B}=3.9$ to represent a dielectric substrate.

To explore the long wavelength limit of the dielectric function of single layer $\mathrm{Sb}$, we make use of the following relation with the imaginary part of the 2D optical conductivity [22]:

$$
\operatorname{Re} \epsilon_{\alpha \alpha}(\mathbf{q}=0, \omega)=1-\frac{\operatorname{Im} \sigma_{\alpha \alpha}(\omega)}{\omega d \epsilon_{B}},
$$

where $d$ is the height of single-layer $\mathrm{Sb}$, which can be approximated as the interlayer distance in its bulk structure $(d=0.36$ $\mathrm{nm})$, and $\operatorname{Im} \sigma_{\alpha \alpha}(\omega)$ is obtained by using the Kramers-Kronig relations from the optical conductivity along the $\alpha$-direction

$$
\begin{aligned}
\operatorname{Re}\left(\sigma_{\alpha \alpha}(\omega)\right)= & -\frac{g_{S}}{\Omega \omega} \int_{\mathrm{BZ}} \operatorname{Im}\left[\sum_{l, l^{\prime}}\left|\left\langle\mathbf{k} l\left|J_{\alpha}\right| \mathbf{k} l^{\prime}\right\rangle\right|^{2}\right. \\
& \left.\times \frac{f\left(E_{\mathbf{k} l}-\mu\right)-f\left(E_{\mathbf{k} l^{\prime}}-\mu\right)}{E_{\mathbf{k} l}-E_{\mathbf{k} l^{\prime}}+\omega+i \delta}\right] d^{2} \mathbf{k},
\end{aligned}
$$

where $\Omega$ is the unit cell surface, and $J_{\alpha}$ is the current operator in the $\alpha$-direction:

$$
J_{\alpha}=-\frac{i e}{\hbar} \sum_{i, j} t_{i j}\left(\mathbf{r}_{j}-\mathbf{r}_{i}\right)_{\alpha} c_{\mathbf{k} i}^{\dagger} c_{\mathbf{k} j}
$$

For isotropic materials such as antimonene, the subscript $\alpha$ in the dielectric function can be ignored as $\epsilon$ is also isotropic and $\epsilon_{x x}=\epsilon_{y y}$.

\section{RESULTS}

\section{A. Dynamical polarization}

The particle-hole excitation spectrum of the $2 \mathrm{D}$ electron gas in single-layer antimonene is defined, as usual, as the region of the energy-momentum space that is available for electron-hole excitations. For noninteracting electrons, it is defined as the region where $\operatorname{Im} \Pi(\mathbf{q}, \omega)$, as given by Eq. (2), is nonzero [21]. Figure 2 shows the dynamical polarization function for different directions of $\mathbf{q}$ (along $\Gamma-\mathrm{M}$ and $\Gamma-\mathrm{K}$ ) and different chemical potentials $\mu$, at $T=300 \mathrm{~K}$. Due to the high number of bands taken into account in the calculation, the spectrum is rich in features with contributions from many interband excitations. Although similar, the dynamical polarization for both directions of the wave-vector features some different excitations. We observe that modifying the chemical potential in Eq. (2) leads to the inclusion, or exclusion, of additional interband electron-hole excitations, resulting in extra low-energy peaks in the dynamical polarization. For a given wave-vector $\mathbf{q}$, we can see that the spectra for different values of $\mu$ present similar structure at high energies, while they are notably different at low energies. The high energy sector of the polarization function $\Pi(\mathbf{q}, \omega)$ stays mostly undisturbed because it is built by interband transitions involving the lower (higher) energy bands of the hole (electron) sectors. For undoped antimonene $(\mu=0)$, only interband transitions are allowed, and therefore $\operatorname{Im} \Pi(\mathbf{q}, \omega)=0$ for frequencies below the band gap $\omega<\Delta[23]$.

When we consider electron or hole doping, we obtain a finite contribution to the spectral weight at low energies, due to activation of intraband electron-hole transitions. These are the processes that dominate the spectrum at long wavelengths, as it is seen for the small wave-vector sector of Figs. 2(a) and 2(d) [see also Figs. 8(b) and 8(c) of the Appendix].

\section{B. Dielectric function and collective modes}

The next step is to consider electron-electron interaction in the spectrum. Within the RPA, the dielectric function of the system $\epsilon(\mathbf{q}, \omega)$ is calculated from Eq. (3), and the corresponding results are shown in Fig. 3. As usual, the zeros of the dielectric function will define the existence and dispersion of collective plasmon modes. There are two distinct regions where $\operatorname{Re}[\epsilon(\mathbf{q}, \omega)]=0$, indicating the existence of several modes. In particular, there is a high-energy plasmon mode at around $\omega \sim 7 \mathrm{eV}$. The exact location of these modes depend on both the chemical potential $\mu$ and the wave-vector $\mathbf{q}$ in the BZ. We must notice that, although this mode corresponds to a zero of the dielectric function, as it can be seen in Fig. 3, it is a mode whose dispersion lies inside the continuum of electronhole excitations: $-\operatorname{Im} \Pi\left(\mathbf{q}, \omega_{\mathrm{pl}}\right)>0$ at the plasmon energy 

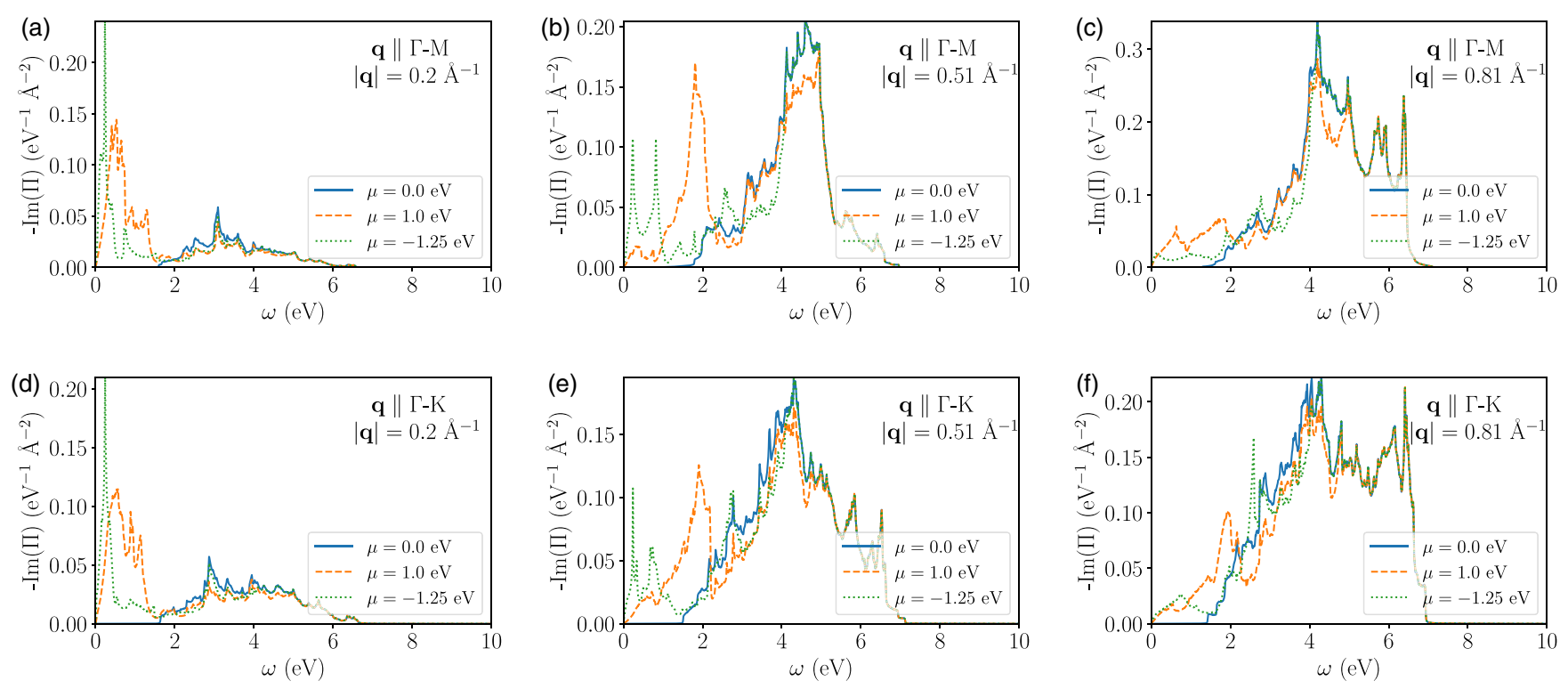

FIG. 2. Imaginary part of the polarization function $\Pi(\mathbf{q}, \omega)$ for different values of wave-vector $\mathbf{q}$ and chemical potential $\mu$. In the top images, $\mathbf{q}$ lies on the $\Gamma-\mathrm{M}$ line in the BZ, while in the bottom images $\mathbf{q}$ lies on the $\Gamma-\mathrm{K}$ line.

$\omega_{\mathrm{pl}}$, what implies that the mode is damped, continuously decaying into electron-hole pairs. Nevertheless, it is a welldefined mode that could be measured experimentally using electron energy loss spectroscopy (EELS) [24], technique that has been successfully applied to study the plasmon spectrum of graphene [25] and other 2D materials [26].

As discussed in the previous section, changing the chemical potential only has a noticeable effect for energy values smaller than $\sim 4 \mathrm{eV}$ [27]. In the presence of finite doping $\mu$ and for small wave vectors, the standard low-energy plasmon mode is recovered. The dielectric function of antimonene in the long wavelength limit is given for different values of the chemical potential, in Fig. 4. The main features of the low-energy spectrum will be discussed in Sec. IV.

\section{DISCUSSION}

In this section, we focus on the low-energy region of the excitation spectrum, which is the most relevant for future experimental probes and possible applications. Figure 5 shows the loss function for hole-doped antimonene with $\omega \leqslant 1 \mathrm{eV}$ and $q \leqslant 0.1 \AA^{-1}$ for different values of $\mu$, including SOC or not in the calculation. Each contour plot is extrapolated from $50 q$ points. The first thing we notice is the existence of an (a)

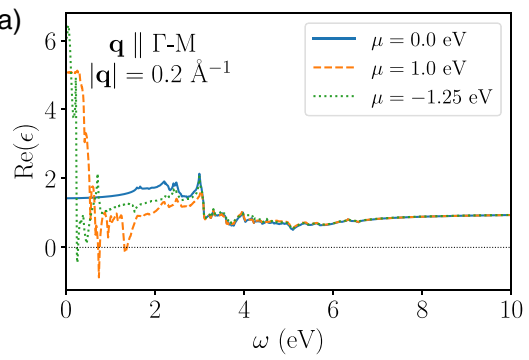

(d)

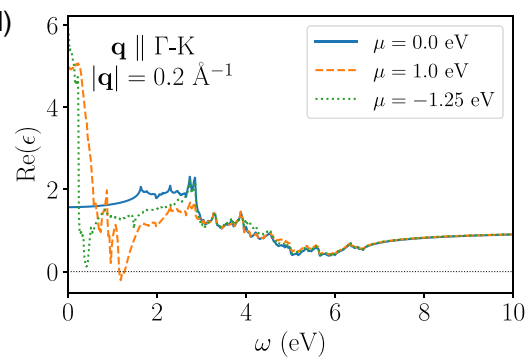

(b)

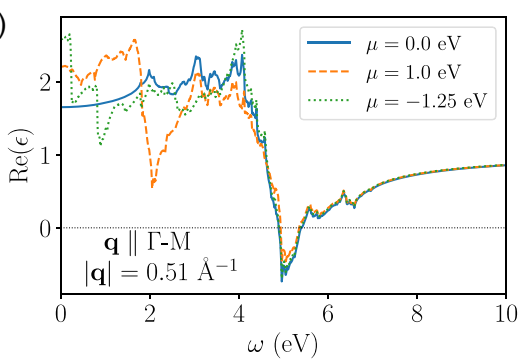

(e)

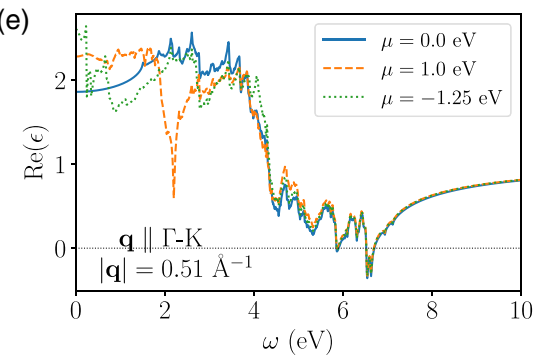

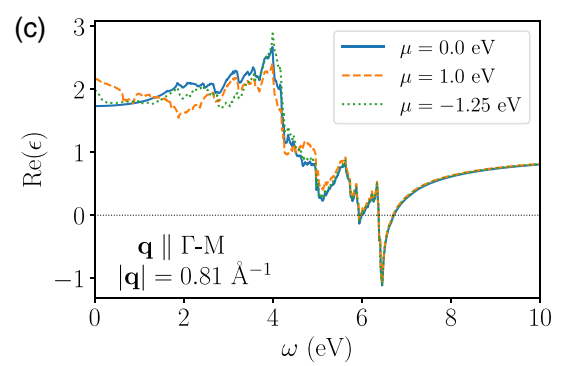

(f)

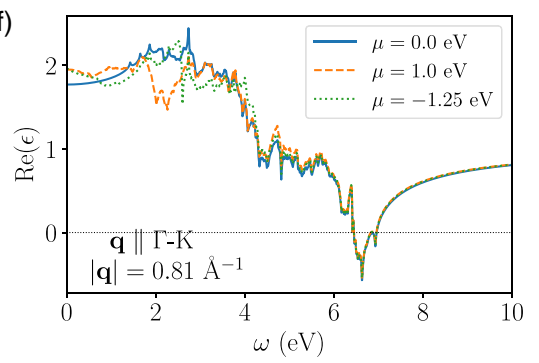

FIG. 3. Real part of the dielectric function $\epsilon(\mathbf{q}, \omega)$ for different wave-vector $\mathbf{q}$ and chemical potential $\mu$. In the top images, $\mathbf{q}$ lies along the $\Gamma-\mathrm{M}$ direction of the BZ, while in the bottom images $\mathbf{q}$ lies along $\Gamma-\mathrm{K}$. For a given $\mathbf{q}$ and $\mu$, the energy of the collective plasmon mode is given by the zeros of the dielectric function. 


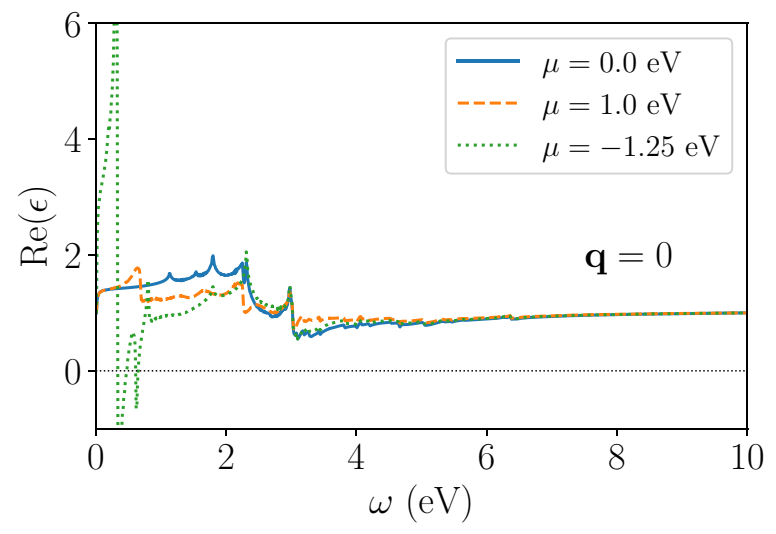

FIG. 4. Real part of the dielectric function $\epsilon(0, \omega)$ for different chemical potential $\mu$, calculated using Eq. (4).

intraband plasmon with a dispersion $\propto \sqrt{q}$. This is expected for any 2D electron gas, and constitutes the standard collective oscillation of carriers due to long-range Coulomb interaction [28]. The dispersion of this mode, at low energies and for $q \rightarrow 0$, is

$$
\omega_{\mathrm{pl}}(q) \approx \sqrt{\frac{2 e^{2} \mu}{\varepsilon_{b}} q+\frac{3}{4} v_{F}^{2} q^{2}},
$$

where $v_{F}=\partial \epsilon /\left.\partial k\right|_{k=k_{F}}$ is the Fermi velocity and $k_{F}=$ $\sqrt{4 \pi n / g}$ is the Fermi wave vector, in terms of the carrier density $n$ and the degeneracy factor $g=g_{s} g_{v}$, where $g_{s}=2$ is the spin degeneracy and $g_{v}=1$ is the valley degeneracy for carriers in a single hole pocket around the $\Gamma$ point of the BZ.

By looking at the loss spectrum for $\mu=-0.75 \mathrm{eV}$ in Fig. 5(d), we can corroborate the existence of a mode whose dispersion responds to Eq. (7). To analyze the effect of SOC,

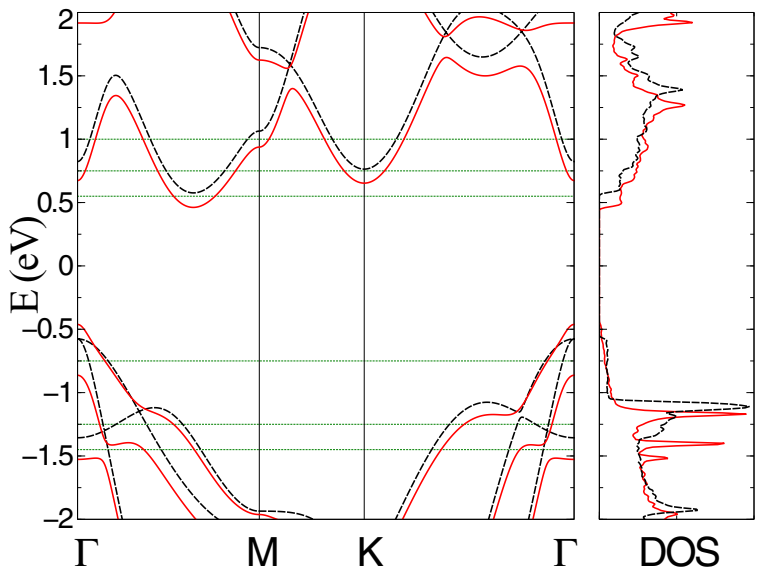

FIG. 6. Comparison of the band structure and density of states of single-layer antimonene with (solid red) and without (dashed black) spin-orbit coupling. Green dotted lines show the values of the chemical potential $\mu$ used in the calculations of the loss function in Fig. 5.

let us compare Figs. 5(a) and 5(d). If SOC is neglected, there are two bands (labeled here as $i=1,2$ ) that are occupied for $\mu=-0.75 \mathrm{eV}$. See Fig. 6 for a clear visualization of the band filling at given $\mu$, for the cases with (solid red) and without (dashed black) SOC. Notice that, if SOC is not considered, these bands are degenerate exactly at the $\Gamma$ point. The loss function for this case, Fig. 5(a), shows two well-defined branches. The branch with larger slope is the plasmon, while the other structure is originated from the interaction between the two intraband single-particle continua associated to bands 1 and 2. The equation for the plasmon in this case has the
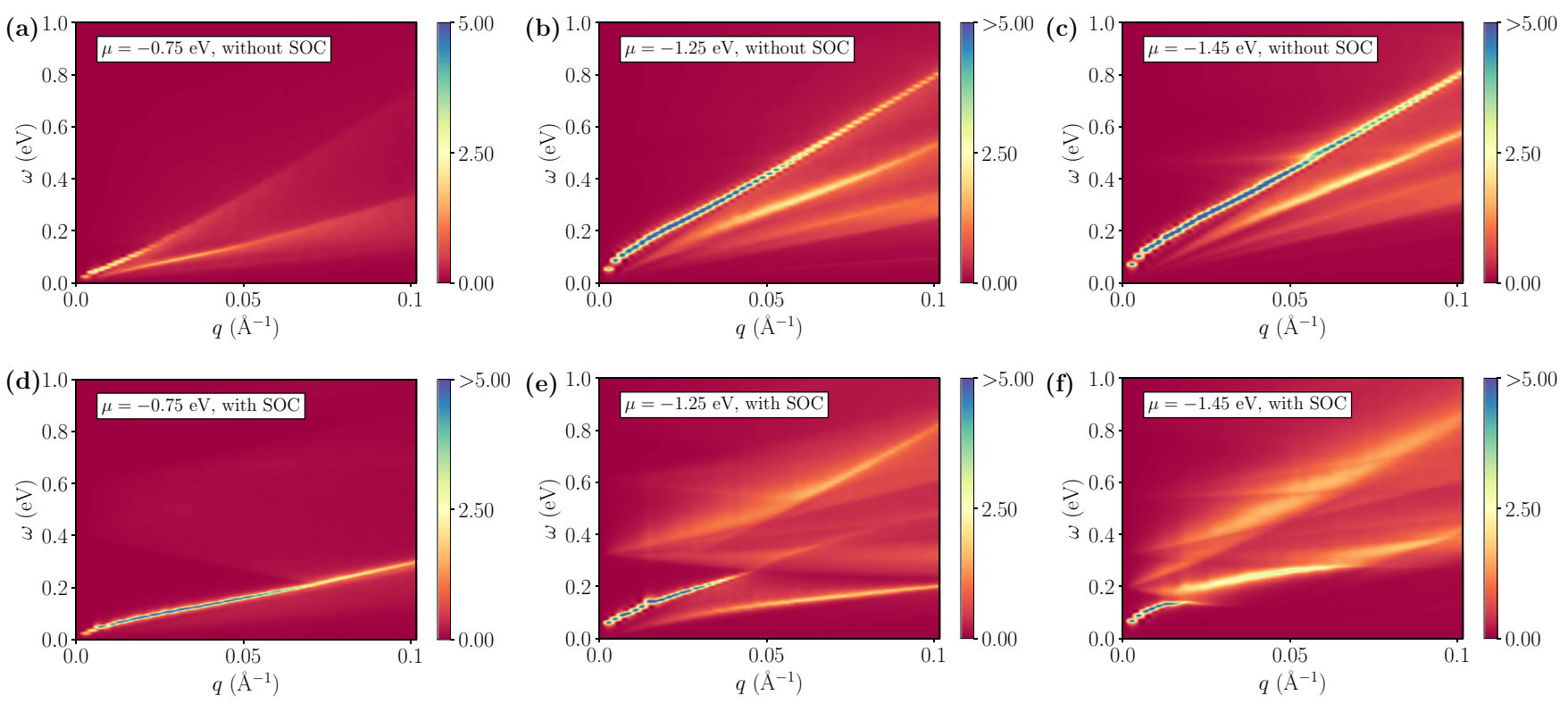

FIG. 5. The loss function $-\operatorname{Im}(1 / \epsilon)$ in the low-energy and wave-vector region of the spectrum. Panels in the first row correspond to $-\operatorname{Im}(1 / \epsilon)$ without SOC, while the second row is with SOC. For each image, the loss function is calculated for $50 q$ points, with fitted points in between. Due to this, some of the figures have a chopped appearance. 

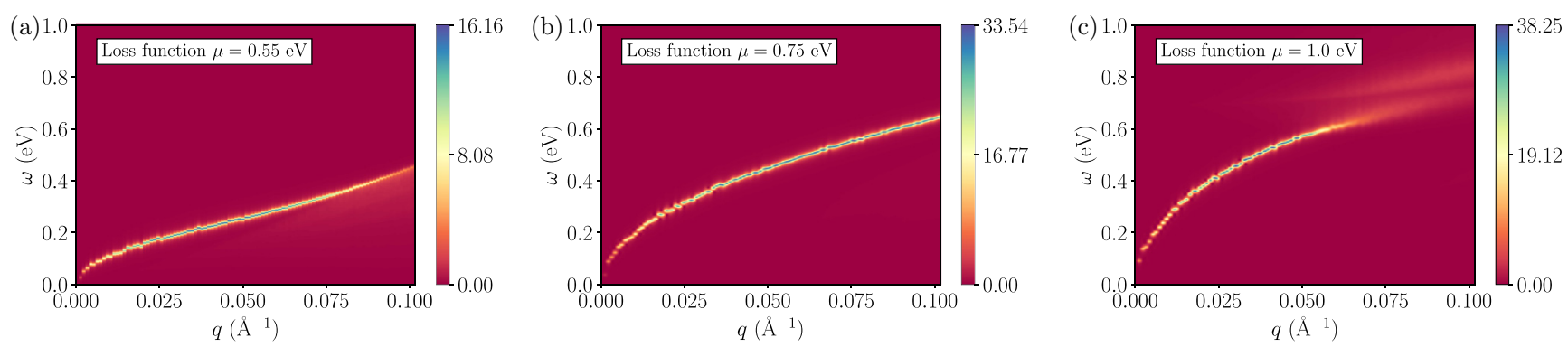

FIG. 7. The loss function $-\operatorname{Im}(1 / \epsilon)$ in the low-energy and wave-vector region of the spectrum for electron doping. For each image, the loss function is calculated for $50 q$ points, with fitted points in between. Due to this, some of the figures have a chopped appearance.

form [29]

$$
\begin{aligned}
& {\left[1-V(q) \Pi_{1}^{0}(\mathbf{q}, \omega)\right]\left[1-V(q) \Pi_{2}^{0}(\mathbf{q}, \omega)\right]} \\
& \quad-V(q)^{2} \Pi_{1}^{0}(\mathbf{q}, \omega) \Pi_{2}^{0}(\mathbf{q}, \omega)=0,
\end{aligned}
$$

where the polarization function of the carriers of band $i=1,2$ can be approximated, in the dynamical long wavelength limit, as [21]

$$
\Pi_{i}^{0}(\mathbf{q}, \omega) \approx \frac{n_{i}}{m_{i}} \frac{q^{2}}{\omega^{2}},
$$

where $n_{i}$ and $m_{i}$ are the corresponding density and effective mass of carriers in pocket $i$. The solution of Eq. (8) gives a plasmon mode with low-energy dispersion

$$
\omega_{\mathrm{pl}}(q) \propto \sqrt{\left(\frac{n_{1}}{m_{1}}+\frac{n_{2}}{m_{2}}\right) q .}
$$

However, the role of SOC has been proven to be very important in antimonene [20], leading to a reconstruction of the band structure that, in particular, lifts the degeneracy between those two bands at the $\Gamma$ point (see Fig. 6). Therefore, a reliable calculation that includes SOC considers, for the same value of $\mu \approx-0.75 \mathrm{eV}$, carriers filling only one subband, with the corresponding reduction (for similar values of the effective masses) in the slope of the dispersion, as it is indeed observed in Fig. 5(d).

Inclusion of SOC is not only necessary to get the correct dispersion relation of the plasmon. The reconstruction of the band structure after consideration of SOC is such that several avoided crossings that occur lead to emergence of new modes in the spectrum, as it can be seen by comparing Figs. 5(b) and 5(e), or Figs. 5(c) and 5(f). The new modes, seen as bright branches in the excitation spectrum, are gapped single-particle resonances associated to interband transitions. The number of these modes is determined by the possible interband transitions from occupied to unoccupied states. Their intensity depend on the DOS at the corresponding energy, which are enhanced for flat bands (saddle points) in the dispersion, associated to the aforementioned avoided crossings in the band structure due to SOC (see lateral panel of Fig. 6 for a comparison of the DOS). Importantly, these interband modes interact with the intraband plasmon, which becomes a source of damping for this mode, an effect that is absent when SOC is neglected. Such effect can be easily seen in Figs. 5(e) and 5(f), where the intraband $\sim \sqrt{q}$ plasmon interact with intersubband continuum, with boundaries $E_{i j} \pm v_{F} q+q^{2} / 2 m$ (assuming equally dispersing parabolic bands), where $E_{i j}$ is the energy separation between bands $i$ and $j$. When the intraband plasmon hits the intersubband continuum, the mode is damped, decaying into interband electron-hole pairs, leading to avoided crossings in the dispersion relation. This effect is reminiscent of the interaction of graphene plasmons with optical phonons [30], and could also be observed with midinfrared transmission measurements, or with EELS experiments, as discussed experimentally [25,26] and theoretically [31-33] for graphene.

We finally consider the case of electron doping. In the lowenergy sector of the conduction band, SOC only leads to some shifts of the band edges, with no qualitative difference with respect to the spectrum without SOC (see Fig. 6). Therefore
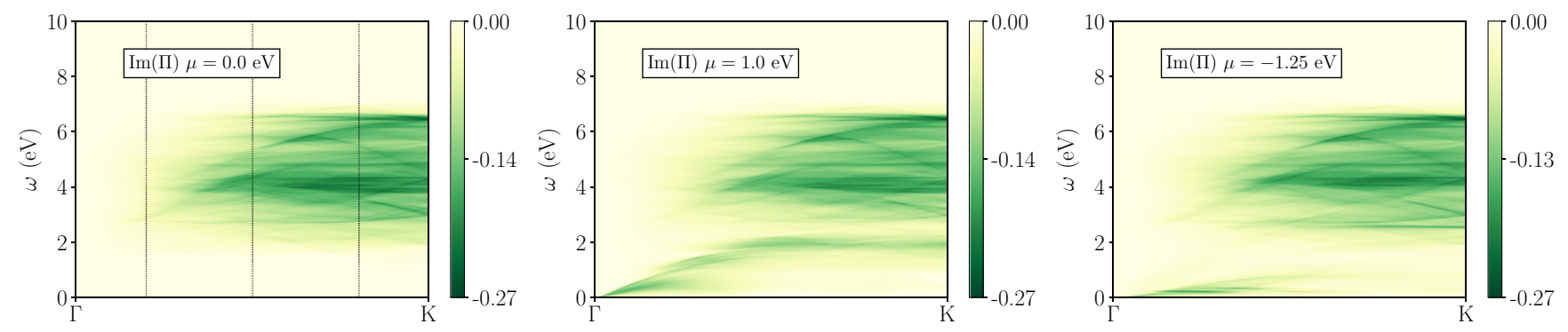

FIG. 8. Electron-hole excitation spectrum of single-layer antimonene. Imaginary part of the polarization function Im $\Pi^{0}(\mathbf{q}, \omega)$ for different values of the chemical potential $\mu$, with $\mathbf{q}$ along the $\Gamma-\mathrm{K}$ line in the BZ. The dotted black lines represent the $\mathbf{q}$ values depicted in the bottom images of Fig. 2. 

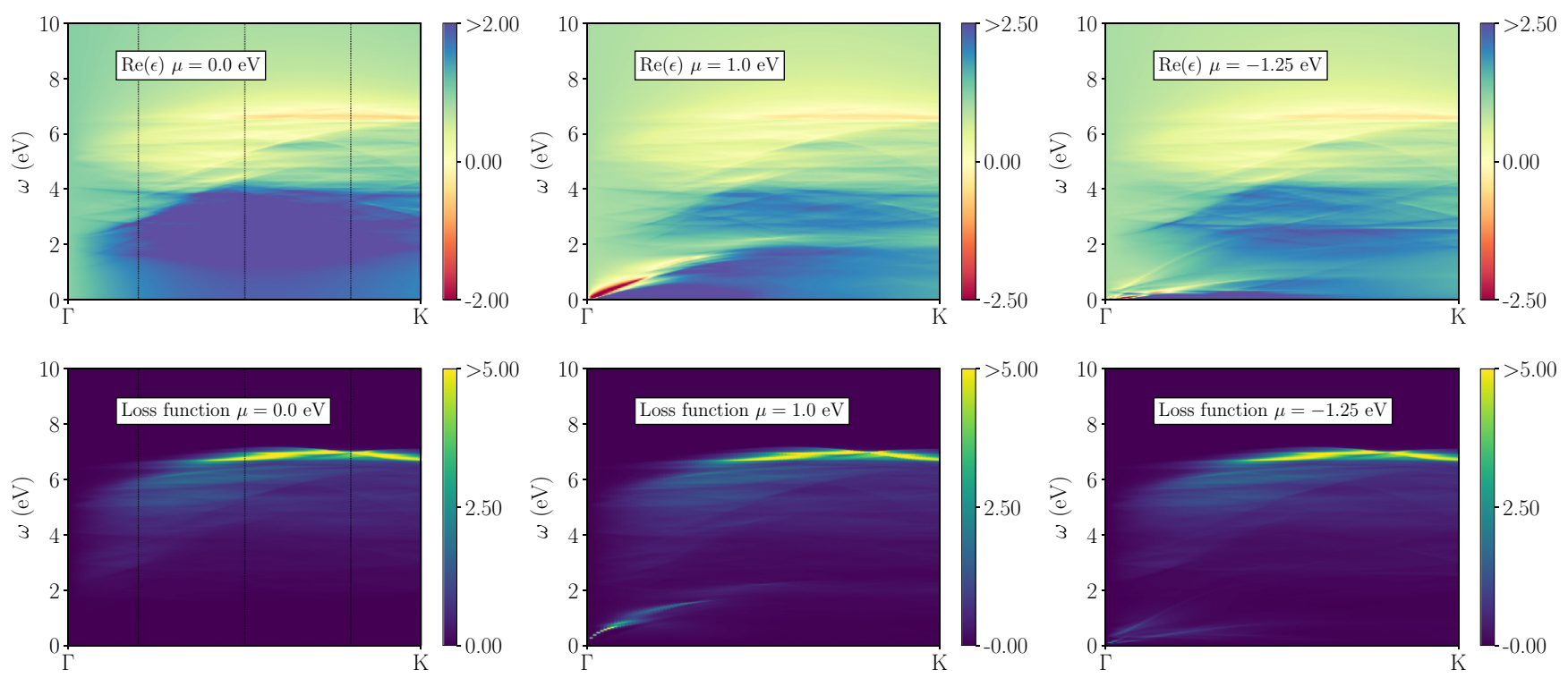

FIG. 9. Real part of the dielectric function $\epsilon(\mathbf{q}, \omega)$ (top panels) and the loss function $-\operatorname{Im}(1 / \epsilon(\mathbf{q}, \omega))$ (bottom panels) for different values of the chemical potential $\mu$, with $\mathbf{q}$ along $\Gamma-\mathrm{K}$ of the Brillouin zone. The black dotted lines indicate the values of $\mathbf{q}$ used in Fig. 3.

we present only the excitation spectrum of antimonene for the electron-doped regime including SOC. The loss function, Fig. 7, shows the existence of a well defined plasmon branch whose slope increases with doping. This is expected because, for a single occupied band, the velocity of the mode grows with $\mu$, Eq. (7). Furthermore, as discussed above, the slope also increases as one starts filling new pockets of the band structure, as given by Eq. (10).

\section{CONCLUSION}

In summary, we have calculated the excitation spectrum for single layer antimony, using a six-orbital TB Hamiltonian that includes SOC. The dielectric function is calculated within the RPA. We obtain a rich spectrum that contains standard plasmons and a set of interband modes originated from the band reconstruction due to SOC. At high energies, the obtained loss functions show a broad peak at $\sim 6 \mathrm{eV}$, associated to the existence of a plasmon, which could be measured by EELS experiments [26]. At low energies, the interaction between the plasmon and the interband single-particle continuum leads to damping of this collective mode. We find avoided crossing features in the dispersion of the modes that could be observed with midinfrared transmission measurements [30].

\section{ACKNOWLEDGMENTS}

R.R. acknowledges financial support from the Spanish MINECO through the Ramón y Cajal program, Grants No. RYC-2016-20663 and No. FIS2014- 58445-JIN. M.I.K. acknowledges financial support from the European Research Council Advanced Grant Program (Contract No. 338957). S.Y. acknowledges financial support from Thousand Young Talent Plan (China). This work is sponsored by NWO Exact and Natural Sciences for the use of supercomputer facilities.

\section{APPENDIX}

To obtain more information about the collective modes, in this Appendix we calculate the loss function, defined as $-\operatorname{Im}(1 / \epsilon)$. Figure 8 shows the noninteracting electron-hole continuum of single-layer antimonene in the $\omega-\mathbf{q}$ plane and Fig. 9 shows the loss spectrum. Notice that, whereas in the dynamical polarization and in the dielectric function, the spectrum consists of many different peaks of similar amplitude, in the loss function there are some modes that clearly dominate. For these, both the imaginary part and the real part of the dielectric function tend to zero, giving rise to well-defined modes in the loss spectrum, which could be directly observed with experimental measurements such as EELS.
[1] P. Ares, J. J. Palacios, G. Abellán, J. Gómez-Herrero, and F. Zamora, Adv. Mater. 30, 1703771 (2018).

[2] P. Ares, F. Aguilar-Galindo, D. Rodríguez-San-Miguel, D. A. Aldave, S. Díaz-Tendero, M. Alcamí, F. Martín, J. Gómez-Herrero, and F. Zamora, Adv. Mater. 28, 6332 (2016).

[3] C. Gibaja, D. Rodriguez-San-Miguel, P. Ares, J. GómezHerrero, M. Varela, R. Gillen, J. Maultzsch, F. Hauke, A. Hirsch, G. Abellán et al., Angew. Chem. Int. Ed. 55, 14345 (2016).
[4] J. Ji, X. Song, J. Liu, Z. Yan, C. Huo, S. Zhang, M. Su, L. Liao, W. Wang, Z. Ni et al., Nat. Comm. 7, 13352 (2016).

[5] T. Lei, C. Liu, J.-L. Zhao, J.-M. Li, Y.-P. Li, J.-O. Wang, R. Wu, H.-J. Qian, H.-Q. Wang, and K. Ibrahim, J. Appl. Phys. 119, 015302 (2016).

[6] X. Wu, Y. Shao, H. Liu, Z. Feng, Y.-L. Wang, J.-T. Sun, C. Liu, J.-O. Wang, Z.-L. Liu, S.-Y. Zhu et al., Adv. Mater. 29, 1605407 (2017).

[7] F. Xia, H. Wang, D. Xiao, M. Dubey, and A. Ramasubramaniam, Nat. Photon. 8, 899 (2014). 
[8] A. Grigorenko, M. Polini, and K. Novoselov, Nat. Photon. 6, 749 (2012).

[9] T. Low, A. Chaves, J. D. Caldwell, A. Kumar, N. X. Fang, P. Avouris, T. F. Heinz, F. Guinea, L. Martin-Moreno, and F. Koppens, Nat. Mater. 16, 182 (2017).

[10] B. Wunsch, T. Stauber, F. Sols, and F. Guinea, New J. Phys. 8, 318 (2006).

[11] E. H. Hwang and S. Das Sarma, Phys. Rev. B 75, 205418 (2007).

[12] M. Polini, R. Asgari, G. Borghi, Y. Barlas, T. Pereg-Barnea, and A. H. MacDonald, Phys. Rev. B 77, 081411 (2008).

[13] T. Stauber, J. Phys.: Condens. Matter 26, 123201 (2014).

[14] T. Low, R. Roldán, H. Wang, F. Xia, P. Avouris, L. M. Moreno, and F. Guinea, Phys. Rev. Lett. 113, 106802 (2014).

[15] F. Jin, R. Roldán, M. I. Katsnelson, and S. Yuan, Phys. Rev. B 92, 115440 (2015).

[16] B. Ghosh, P. Kumar, A. Thakur, Y. S. Chauhan, S. Bhowmick, and A. Agarwal, Phys. Rev. B 96, 035422 (2017).

[17] S. Dai, Z. Fei, Q. Ma, A. Rodin, M. Wagner, A. McLeod, M. Liu, W. Gannett, W. Regan, K. Watanabe et al., Science 343, 1125 (2014).

[18] R. Roldán, L. Chirolli, E. Prada, J. A. Silva-Guillén, P. San-Jose, and F. Guinea, Chem. Soc. Rev. 46, 4387 (2017).

[19] S. Zhang, Z. Yan, Y. Li, Z. Chen, and H. Zeng, Angew. Chem. 127, 3155 (2015).

[20] A. N. Rudenko, M. I. Katsnelson, and R. Roldán, Phys. Rev. B 95, 081407(R) (2017).
[21] G. F. Giuliani and G. Vignale, Quantum Theory of the Electron Liquid (Cambridge University Press, Cambridge, 2005).

[22] J. S. Seybold, Introduction to RF Propagation (John Wiley \& Sons, Inc., Hoboken, NJ, 2005).

[23] For a more complete visualisation of the excitation spectrum, in Fig. 8 of the Appendix we show a density plot of $\operatorname{Im} \Pi(\mathbf{q}, \omega)$ in the $\omega-\mathbf{q}$ plane.

[24] R. F. Egerton, Electron Energy-Loss Spectroscopy in the Electron Microscope (Springer Science \& Business Media, New York, NY, 2011).

[25] T. Eberlein, U. Bangert, R. R. Nair, R. Jones, M. Gass, A. L. Bleloch, K. S. Novoselov, A. Geim, and P. R. Briddon, Phys. Rev. B 77, 233406 (2008).

[26] A. Politano, G. Chiarello, and C. Spinella, Mater. Sci. Semicond. Process. 65, 88 (2017).

[27] See also Fig. 9 of Appendix for a density plot of $\operatorname{Re} \varepsilon(\mathbf{q}, \omega)$ in the $\omega-\mathbf{q}$ plane.

[28] F. Stern, Phys. Rev. Lett. 18, 546 (1967).

[29] S. Das Sarma, Phys. Rev. B 29, 2334(R) (1984).

[30] H. Yan, T. Low, W. Zhu, Y. Wu, M. Freitag, X. Li, F. Guinea, P. Avouris, and F. Xia, Nat. Photon. 7, 394 (2013).

[31] S. Yuan, R. Roldán, and M. I. Katsnelson, Phys. Rev. B 84, 035439 (2011).

[32] D. Novko, V. Despoja, and M. Šunjić, Phys. Rev. B 91, 195407 (2015).

[33] I. Kupčić, G. Nikšić, Z. Rukelj, and D. Pelc, Phys. Rev. B 94, 075434 (2016). 